\title{
In vivo modulatory responses of cardiovascular system to ethanolic extract of the root bark of securidaca longipenduncalata in albino rats
}

\author{
Salami $\mathrm{HA}^{1 *}$, Adelaiye $\mathrm{AB}^{2}$, Tukur $\mathrm{MA}^{1}$, Olorunsola $\mathrm{KV}^{2}$ and $\mathrm{Ayo} \mathrm{JO}^{3}$ \\ ${ }^{1}$ Department of Human Physiology, University of Maiduguri, Nigeria. \\ ${ }^{2}$ Department of Human Physiology, University of Abuja, Nigeria. \\ ${ }^{3}$ Department of Veterinary Physiology and Pharmacology, Ahmadu Bello University, Zaria, Nigeria.
}

\begin{abstract}
In vivo evaluation of modulatory responses of Ethanolic extract of the root bark of Securidaca longipendunculata (EESL) in rats on cardiovascular system was monitored. Intravenous, intraperitoneal and electrocardiograph were compared after EESL injection. The intravenous and intraperitoneal produced a dose-dependent gradual decrease in mean arterial blood pressure which is more pronounced in intravenous than intraperitoneal root of administration. The decrease was statistically significant $(\mathrm{P}<0.05)$ at cumulative dose of $36 \mathrm{mg} / \mathrm{kg}$. The electrocardiographic record showed a gradual alteration with time on the electrocardiogram. The initial hearth beat was $300 \pm 0.00$ beat per minute. EESL significantly $(p<0.05)$ decreased heart rate from $300 \pm 0.00$ to $49.02 \pm 0.39$ at about 122 minutes after administration, also the amplitude was significantly $(\mathrm{P}<0.001)$ raised from $10.42 \pm 0.24 \mathrm{mV}$ (initial amplitude) o $21.60 \pm 0.67$ around 122 minutes after administration. The ST-segment was elevated and QRS complex was predominantly negative towards the death of animal. This finding suggests that EESL lowers blood pressure in rat and intravenous effect is more pronounced than intraperitoneal.
\end{abstract}

\section{Introduction}

Cardiovascular (CVS) regulation is complex, it involves either regulation of heart or vessels, and in some cases both of these organs must be regulated. The commoner disease associated with cardiovascular system worldwide is high blood pressure (Lozarno et al., 2012). The proportion of high blood pressure (Hypertension) burden has significantly increased from about 4.5 percent (nearly one billion adult) in 2000 (Kearney et al., 2005) to 7 percent in 2010 (Lim, 2010) which make hypertension the single most important cause of morbidity and mortality globally (Kizhakekuttu and Widlansky, 2010). Currently the worldwide burden of hypertension is reported to be greatest in low and middle-income countries where about 1 in every 5 of the adult population is affected (Seedat $e t$ al., 2000). More so, significant proportion of hypertensive patient do not respond to modern medication while high cost of modern medication, side effects, accessibility and availability particularly in rural setting compound these problems. Because of undesired side effect of modern antihypertensive drugs (Susalit et al., 2011; Wang and Xiong 2012) and reduced patient compliance to conserve more than a pill per day (August 2004) this patient especially those in rural area seek alternative approaches such as herbal remedies. The use of herbal remedies has significantly increased over the decades (Frishman et al., 2009) because it is cheaper with fewer undesired side effects (Susalit et al., 2011; Tobassum and Ahmed 2011).

One of the local herbal remedies used in this region for high blood pressure control is Securidaca longipendunculata in the North Eastern of Nigeria. Previous studies on this plant have indicated that the root extract of Securidaca longipendunculata possessed a dose-dependent inhibition of pressure effect of noradrenaline on the arterial blood pressure and heart rate (Ojewale et al., 2000), while the in vitro effect of the crude extract on isolated rabbit heart preparation showed a dual effect on heart rate and coronary circulation (Salami et al 2016). Generally, since the mechanisms involve in the regulation of cardiovascular system are complex, the work is design to monitor system changes in response to administration of crude extract EESL using electrograph and physiograph with a view to understand the mechanism of action of Securidaca longipendunculata.

\section{Methods}

The fresh root of Securidaca longipedunculata were purchased from an herbalist. The plant was verified by an herbalist, Malam Abubakar Adamu and was found to be identical with the specimen samples deposited at the herbarium of the Laboratory of Vegetal Biology, University of Maiduguri, Maiduguri. A voucher specimen was also deposited at this herbarium.

\section{Extraction}

After collection, the root was washed, and the bark removed and then air-dried at room temperature. The dried powder ( $400 \mathrm{gm})$ of the root bark was extracted in a Soxhlet apparatus with $60 \% \mathrm{v} / \mathrm{v}$ ethanol.

Correspondence to: Salami HA, Department of Human Physiology, University of Maiduguri, Nigeria

Key words: Cardiovascular Regulation, Hypertension, Securidaca longipendunculata, Intravenous, IntraPeritonuem, Electrocardiogram

Received: March 01, 2018; Accepted: March 15, 2018; Published: March 19 , 2018 
The final extract was evaporated in vacuum and the dry residue was kept at $4^{\circ} \mathrm{C}$ until used. Weighed samples of the extracts were then used to make serial dilution of desired concentrations.

Twenty (20) Adult Wistar rats of both sexes weighing between 150 - $250 \mathrm{~g}$ and were used in this study. The animals were bred in the animal house, College of Medicine, University of Maiduguri, Rats were chosen in preference to rodents for heart experiment because their heart and cardiac circulation more closely resemble those of humans. Animals were acclimatized under standard laboratory conditions and normal photoperiod (12-hour light: dark cycle) for 7 days. Commercial pellet diet and water were provided ad-libitum.

Male Wistar rats (250-300 g) were anaesthetized with a 50-50 mixture of $25 \%$ urethane and $1 \%$ alphachloralose at a dose $5 \mathrm{mg} /$ $\mathrm{kg}$. Tracheotomy was performed to insert endotracheal tube. Right femoral artery and femoral vein were cannulated. The catheter of the femoral artery was connected to a pressure transducer (Grass FT 03) via three-way stopcock was inserted for recording arterial blood pressure while femoral vein cannula was used for drug administration. After cannulation, the rats were injected with heparin $6001 \mathrm{U} / \mathrm{kg}$ body weight to prevent blood clotting. A period of 30 minutes was allowed for the animal to stabilize. The animal was then injected with 0.1-0.5 ml saline $0.9 \% \mathrm{NaCl}$, the same volume of extract of Securidaca longipendunculata used. The arterial blood pressure was allowed to stabilize at a level where no fluctuations observed between injections. The changes in blood pressure recorded as mean arterial blood pressure and heart rate before and after each extract injection were recorded.

\section{Electrocardiographic study}

Adult wistar rats weighing 200-250 g were used. They were anaesthetized with $6 \%$ sodium penthobabitone at the dose $0.1 \mathrm{ml} / 100 \mathrm{~g}$ body weight. The electrocardiographic leads were implanted into the paws of the arms and legs. The animals were allowed $30 \mathrm{~min}$ for blood pressure and the electrocardiographic tracing to stabilize. Acute dose $(45 \mathrm{mg} / \mathrm{kg})$ of extract was then given intraperitoneally and electrocardiographic changes were monitored for 4 hours. The QT interval, QRS complex, the ST segment and the Q-wave were measured.

\section{Statistical analysis}

The data obtained were subjected to statistical package SPSS version 20.0 for analysis. Values were expressed as mean \pm SEM. Data subjected to one-way analysis of variance (ANOVA). Values of $\mathrm{P}<0.05$ were considered significant.

\section{Results}

The results showed that EESL produced dose-dependent decrease in mean arterial blood pressure and dose-dependent increased in the heart rate, except at the dose of $20 \mathrm{mg} / \mathrm{kg}$, when a decrease in heart rate was recorded in rats that received EESL intravenously (Figures 1 and 2). Mean arterial blood pressure decreased from $6.39 \pm 9.61 \%$ at the cumulative concentration of $5 \mathrm{mg} / \mathrm{kg}$ to $69.95 \pm 2.09 \%$ at the cumulative concentration of $20.0 \mathrm{mg} / \mathrm{kg}$, whereas the heart rate was increased from $9.2 \pm 11.5 \%$ at a dose of $1.0 \mathrm{mg} / \mathrm{kg}$ to $30.45 \pm 8.53 \%$ at cumulative dose of $18.5 \mathrm{mg} / \mathrm{kg}$. At the cumulative dose of $38.5 \mathrm{mg} / \mathrm{kg}$ the heart rate was significantly decreased, $(\mathrm{P}<0.05)$ by $27.15 \pm 3.18 \%$ in animals with intravenous administration.

The pattern of responses of blood pressure and heart rate to the intraperitoneal administration of EESL are shown in figures 1 and 2. EESL produced a dose-dependent decrease in mean arterial blood

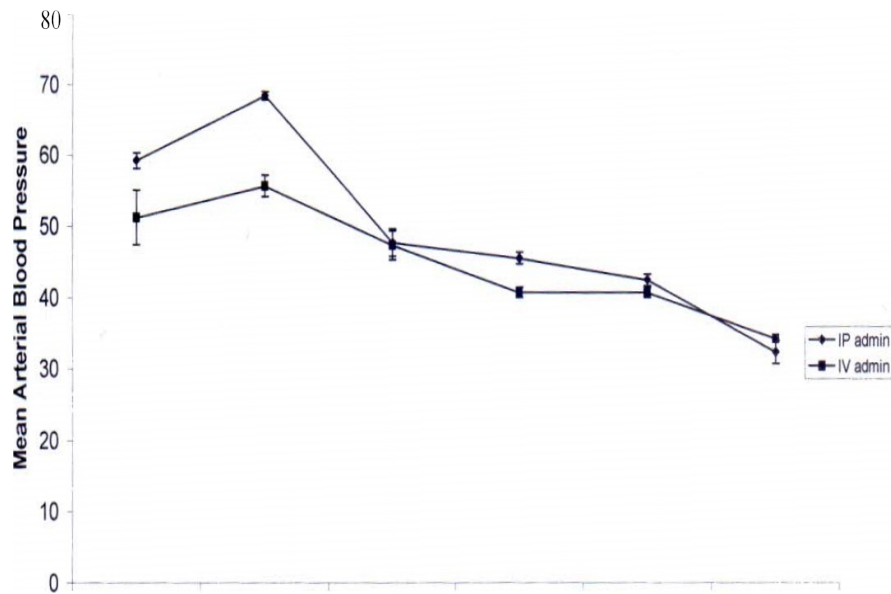

Figure 1. Comparative effect of intravenous and intraperitoneal administration of the extract of Securidaca longipendunculata on mean arterial blood pressure of anaesthetized rats. $\mathrm{IP}=$ Intraperitoneal $\quad \mathrm{IV}=$ Intravenous

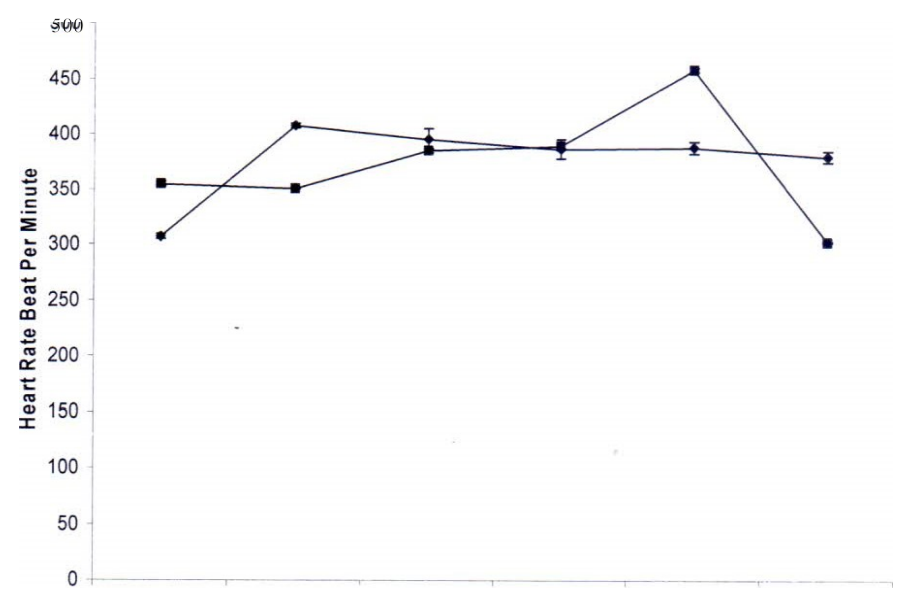

Figure 2. Comparative effect of intravenous and intraperitoneal administration of the extract of Securidaca longipendunculata on heart rate of anaesthetized rats. $\mathrm{IP}=$ Intraperitoneal IV= Intravenous

pressure and a dose-dependent increased in the heart rate The extract decreased mean arterial blood pressure from $19.8 \pm 2.39 \%$ at the cumulative dose of $3.0 \mathrm{mg} / \mathrm{kg}$ to $58.82 \pm 1.10 \%$ at a cumulative dose of $56 \mathrm{mg} / \mathrm{kg}$ respectively The extract on the other hand, has dual effect on heart rate with the initial dose $(1 \mathrm{mg} / \mathrm{kg})$ producing percentage increase in heart rate of $25.20 \pm 1.840 / 0$, followed by a gradual decrease in percentage increased tachycardia $(22.84 \pm 0.850 / 0,21.15 \pm 0.930 / 0$, $21.36 \pm 0.55 \%, 19.94 \pm 0.62 \%$ and $11.31 \pm 2.430 / 0)$ at the doses of $1.0 \mathrm{mg}$, $2.0 \mathrm{mg}, 4.0 \mathrm{mg}, 5.0 \mathrm{mg}, 7.5 \mathrm{mg}$, and $10.0 \mathrm{mg} / \mathrm{kg}$, respectively. The gradual decrease was not significant $(\mathrm{P}>0.05)$ except at a dose $10 \mathrm{mg} / \mathrm{kg}$. At cumulative doses of $44.5 \mathrm{mg} / \mathrm{kg}$ and $56.5 \mathrm{mg} / \mathrm{kg}$, a significant $(\mathrm{P}<0.05)$ sharp increase $(25.12 \pm 1.42 \%$ and $32.83 \pm 1.370 / 0$, respectively) was recorded. Comparable effect of EESL administered intraperitoneally and intravenously showed that the intravenous rout produced more significant percentage decrease in mean arterial blood pressure than the intraperitoneal route and the animal survived for only twentythree minutes. However, a gradual rise in percentage increase in the heart rate was recorded in both intravenous and intraperitoneal administration; except at cumulative dose of $38.5 \mathrm{mg} / \mathrm{kg}$ dose, when the intravenous route of administration induced a sharp decrease in percentage of tachycardia. 
Table 1. Electrocardiographic Changes associated with Securidaca longipedunculata Administration.

\begin{tabular}{|c|c|c|c|c|c|c|c|c|c|}
\hline & Zero time & $2 \mathrm{~min}$ & $6 \mathrm{~min}$ & $12 \mathrm{~min}$ & $22 \mathrm{~min}$ & $47 \mathrm{~min}$ & $77 \mathrm{~min}$ & $92 \mathrm{~min}$ & $122 \mathrm{~min}$ \\
\hline Amplitude & $10.42 \pm 0.24$ & $9.00 \pm 0.31$ & $10.20 \pm 0.37$ & $12.20 \pm 0.20$ & $13.00 \pm 0.00$ & $16.20 \pm 0.48$ & $15.80 \pm 0.58$ & $14.80 \pm 0.37$ & $21.60 \pm 0.67$ \\
\hline Rate/mins & $300 \pm 0.00$ & $280 \pm 12.24$ & $235.71 \pm 8.75$ & $208.92 \pm 5.35$ & $188.57 \pm 15.74$ & $155.97 \pm 10.99$ & $156.64 \pm 4.04$ & $133.42 \pm 6.68$ & $49.02 \pm 0.39$ \\
\hline QRS wave & Positive & Positive & Positive & Positive & Negative & Negative & - & - & - \\
\hline T-wave & Normal & Normal & Normal & Normal & High & High & High & High & - \\
\hline S-T & Online & Online & Online & Online & High & High & High & High & - \\
\hline$P$ & Present & Present & Present & Present & Present & Present & Present & Present & Absent \\
\hline
\end{tabular}

Electrocardiographic tracing showed gradual alteration on electrocardiogram with time. This alteration ranged from mild to severe until death of the animal occurred (Table 1 and Figures 3 and 4 ). The base-line value of the heart rate was $300 \pm 0.00$ beat per minute. Following administration of EESL, a gradual and significant $(\mathrm{P}<0.001)$ decrease in the heart rate values $(250 \pm 0.00$ beat $/ \mathrm{min} 214.28 \pm 0.00$ beat $/ \mathrm{min}, 188.57 \pm 15.74$ beat $/ \mathrm{min}, 152.04 \pm 9.37$ beat $/ \mathrm{min}, 156.66$ $\pm 4.02 \mathrm{beat} / \mathrm{min}, 113.42 \pm 6.68 \mathrm{beat} / \mathrm{min}$, and $49.02 \pm 0.39$ beats per minute) occurred at $12 \mathrm{~min}, 22 \mathrm{~min}, 47 \mathrm{~min}, 77 \mathrm{~min}, 92 \mathrm{~min}$ and at 122 min, when the animal died.

On the other hand, gradual and significant increase $(\mathrm{P}<0.001)$ in the amplitude of contraction with time was observed on the electrocardiogram. The initial amplitude recorded was $10.40 \pm 0.24$ $\mathrm{mV}$. This value doubled $(21.60 \pm 0.67 \mathrm{mV})$ few minutes before the animal's death. The QRS complex was positive for $22 \mathrm{~min}$, after which it was predominantly negative with increase in voltage. Elevation of ST segment was first recorded at $22 \mathrm{~min}$. This was also accompanied with J-wave at 92 minutes of the recording. The P-wave was normal except at 92 and $122 \mathrm{~min}$, when it was absent.

\section{Discussion}

The effect of intravenous and intraperitoneal administration of EESL in Wistar rats were compared. This study showed that EESL administration intraperitoneally, produced dose-dependent hypotension and cardio-acceleratory effect. The same observation was made with intravenous administration. The cardio-accelerating effect comparably less than the effect on the blood vessels. Similar results were obtained with nifedipine in isolated rabbit heart; that is, high coronary flow with no significant depression of heart rate (Salami et al., 2016). The hypotensive effect of the extract was more pronounced and rapid with high percentage increase in heart rate observe in rat that received the extract intravenously. At high doses, severe hypotension was accompanied with depression of heart rate suggesting early death, and total cumulative doses received by this group of rats were lower than those received by groups intraperitoneally administered with the extract.

It is speculated that the effect of the extract on heart in the group that received the extract intraperitoneally may be brought about by compensatory sympathetic reflexes, resulting from the extract-induced decrease in blood pressure, or Sino-reflex mechanism (a rise in the arterial blood pressure leading to a decrease in the heart rate and viceversa). It is believed that the less pressure in Sino-sensitive area will augment increase in heart rate. These results were consistent with the findings of other studies (Sofola and Feyi, 1981; Looaresuwan et al., 1986; Riou et al., 1988). However, the increased heart rate might have not been enough to cope for rapid decrease in blood pressure observed at high doses. This may explain the cause of animals' death. Similar studies have attributed the cause of death to the hypotensive effect of EESL caused by inhibition of pressure effect of noradrenaline on the

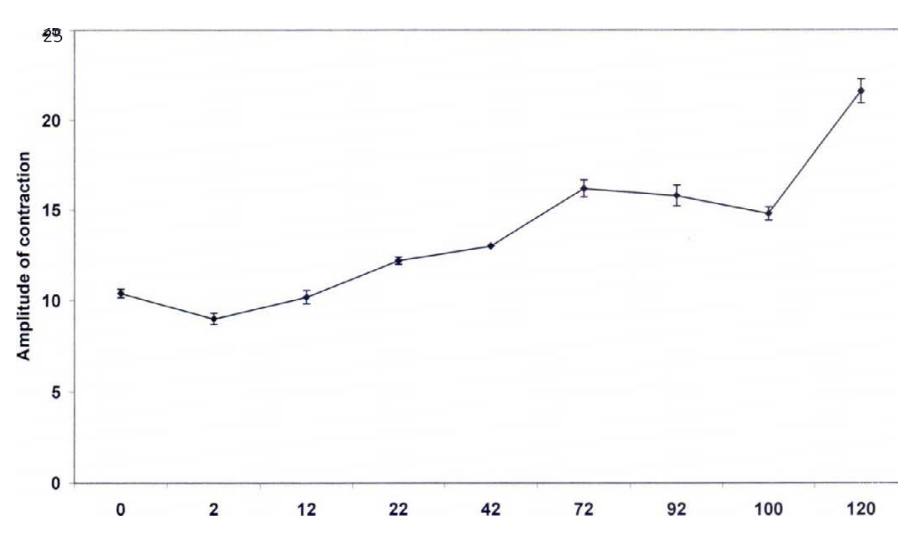

Figure 3. Effect of ethanol extract of Securidaca longipedunculata on amplitude as recorded on the Electrocardiogram.

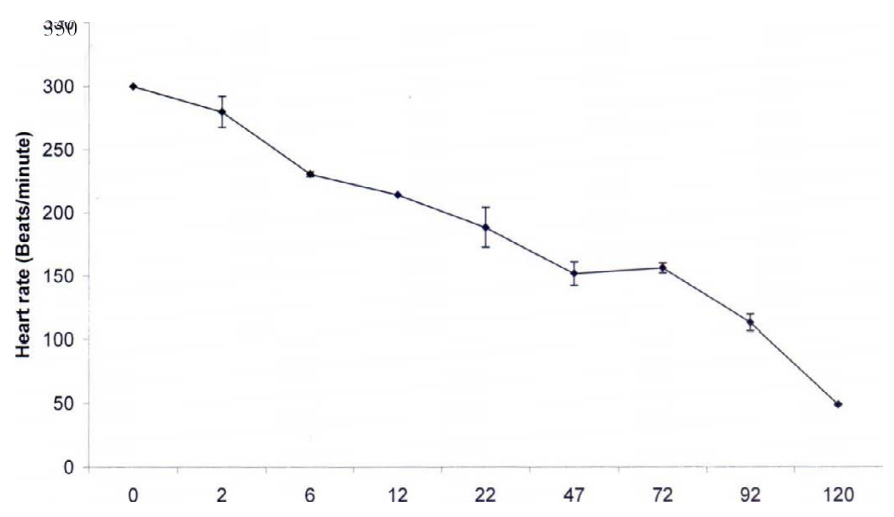

Figure 4. Effect of ethanol extract of Securidata longipedunculata on heart rate as recorded on Electrocardiogram.

arterial blood pressure (Ojewole et al., 2000). This finding suggests that cardioactive effect of the extract resulted probably from increased myocardial calcium ion concentration because the invitro effect of this extract on isolated heart revealed the use of nifedipine to block its effect on coronary flow (Salami et al., 2016). More so an increase inward calcium concentration and binding was reported to be proportional to myocardial contractility and therefore serve the basis for using digitalis drug to treat congestive heart failure (Jian and Zijian, 2008), therefore the EESL action in vivo, at least in part, may be through digitalis-like effect.

Electrocardiographic findings showed that the extract, though did not affect sinus rhythm, increased amplitude of the waves, and sinus bradycardia was observed before the death of the animals. These findings support the observation in the previous study on isolated rabbit heart and in-vitro experiment. The increase in the force of contraction seen in isolated heart preparation (Salami et al., 2016) is also translated on electrocardiogram in the form of high amplitude. The increase in 
amplitude may be seen as evidence of increased intracellular calcium brought about by EESL.

The maximal ST segment elevation was observed before the animal's death. Elevation of ST segment has been described as a sign of inadequate oxygen supply to the heart, mainly due to coronary constriction or embolism (Hiramatsu et al., 2001). Abnormal ST segment was also observed to be associated with gradual increase in negativity of QRS complex, an appearance described in some studies as an evidence of acute anterior myocardial oxygen insufficiency. This suggests the evidence of a decrease in coronary flow obtained in the isolated heart. A decrease in coronary flow has been attributed to be the cause of myocardial infarction. Elevated ST segment observed in the present study indicates the part of the cardiac muscle affected; anterior damage showed in the V leads and inferior damage in leads III and VF. ST segment was either depressed or elevated.

Generally, the appearance of abnormal depression Q-wave recorded on the ECG is been attributed to myocardial ischaemia. The finding is consistent with the appearance of abnormal depressed Q-wave observed on the ECG in this work and can also be attributed to myocardial ischaemia. This result was supported by the study of Sztajzed and Urban (2000), who described a depressed Q-wave as a sign of myocardial ischaemia, and in most cases is associated with ST segment elevation. Other studies suggested that the presence of transient Q-wave depression may identify patients with a potentially great degree of myocardium at risk (Raitt et al., 1995; Willis et al., 2004).

It is therefore, concluded that electrocardiographic studies support our view that the extract induced coronary constriction as supported by the following facts:

\section{1) Elevation of ST segment at lead III Depressed Q wave \\ 2)Increased amplitude of contraction}

3)Sinus bradycardia and cardiac arrest.

In conclusion, these studies obtained so far demonstrated that ethanol extract of Securidaca longipendunculata induce hypotension and tachycardia. The mechanism of action of SL may be similar to digitalis drugs in the treatment of congestive heart failure through calcium channel therefore justified herbalist use of plant for controlling hypertension.

\section{References}

1. Augusti KT, Jose R, Sajitha GR, Augustine P (2012) A rethinking on the benefits and drawbacks of common antioxidants and proposal to look for the antioxidants in allium products as ideal agents: a review. Indian Journal Clinical Biochemistry 27: 6-20. [Crossref]

2. Frishman WH, Beravol P, Carosella C (2009) Alternative and complementary medicine for preventing and treating cardiovascular disease. Disease Mon 55: 121-192. [Crossref]
3. Hiramatsu S, Morimoto S, Kato S (2001) Transient ventricular wall wall thickening in acute myocarditis: a serial echocardiographic and histological study. Japan Circulation Journal 65: 863-866 [Crossref]

4. Jian T, Zijian X (2008) The Na-K ATpase Calcium Signaling Microdomains. Physiology Reviews 23: 205-211 [Crossref]

5. Kearney PM, Whelton M, Reynolds K, Muntner P, Whelton PK, (2005) Global burden of hypertension: analysis of worldwide data. Lancet 365: 217-23. [Crossref]

6. Kizhakekuttu TJ, Widlansky ME (2010) Natural antioxidants and anti hypertention: Promises and Challenges. Cardiovascular therapy 28: e20-e32. [Crossref]

7. Lim SS, Vos T, Flaxman AD, Danaei G, Shibuya K, Adair-Rohani H (2012) A comparative risk assessment of burden of disease and injury attributable to 67 risk factors and risk factor clusters in 21 regions: a systematic analysis for the global burden of disease study. The Lancet 380: 2224-2260. [Crossref]

8. Looareesuwan S, White N, Chanthavanichi P, Edward G, Nicholl D, Bunch C, Warrel D (1986) Cardiovascular toxicity and distribution kinetic of intravenous chloroquine. British journal of clinical pharmacology 22: 31-36. [Crossref]

9. Lozarno R, Naghavi M, Foreman K, Lim S, Shibuya K, Aboyan V (2012) Global and regional mortality from 235 causes of death for 20 age groups in 1990 and 2010 a systemic analysis for global burden of disease study 2010. The Lancet 380: 2095-2128. [Crossref]

10. Ojewole JA, Ilesanmi RS, Olayiwola G (2000) Neuromuscular and cardiovascular properties of Securidaca longipendunculata, Nigerian Journal of Natural Product and Medicine.

11. Raitt MH, Maynard C, Wagner GS (1995) Appearance of abnormal Q-wave early in the course of acute myocardial infarction implication for efficacy of thrombolytic therapy. Journal American College of Cardiology 25: 1084-1088. [Crossref]

12. Riou B, Barriot P, Rimaiho A, Band FJ (1988) Treatment of severe chloroquine poisoning. Northern England Journal of Medicine 318: 1-6. [Crossref]

13. Salami HA (2016) European Journal of Biomedical and Pharmaceutical Sciences: Effect of crude extract of Securidaca longipedunculata on isolated rabbit heart: 3: 103-108.

14. Seedat YK (2000) Hypertension in developing nations in sub-saharan Africa. Journal of Human Hypertension 11: 739-4.

15. Smith R (2012) Why a macroeconomic perspective is critical to the prevention of noncommunicable disease. Scince 337: 1501-1503. [Crossref]

16. Sofola OA, Feyi (1981) The effect of chronic chloroquine toxicity on blood pressure of rats. Journal of Tropical Medicine and Hygiene 249-252.

17. Susalite E, Agus N, Effendit I, Tjandrawinata RR, Mofiarny D, Perrinjaquetmoccetti T (2011) Olive (alea europaeal) leaf extract effective in patients with stage 1 hypertension comparison with captopril. Phytomedicine 18: 251-258 [Crossref]

18. Sztajzel J, Urban P (2000) Early and late Q wave regression in the setting of acute myocardial infarction. Heart 83: 708-710. [Crossref]

19. Tabassum M, Ahmad F (2011) Role of natural herbs in the treatment of hypertension. Pharmacognosy Review 5: 30-40 [Crossref]

20. Wang J, Xiong X (2012) Outcome measures of Chinese herbal medicine for hypertension: an overview of systematic reviews. Evidence based complement alternative medicine: 697237 . 10. 1155/697237

21. Willis H, Leonard LS, Ellen E, Pinnow MS, Joseph Lindsay (2004) The accuracy of electrocardiographic Q-wave for the detection of prior myocardiac infarction as assessed by novel standard of reference. Clinical Cardiology 27: 97-100.

Copyright: (C2018 Salami HA. This is an open-access article distributed under the terms of the Creative Commons Attribution License, which permits unrestricted use, distribution, and reproduction in any medium, provided the original author and source are credited. 